\title{
Low Complexity Frequency Offset Estimation for FMCW Radar
}

\author{
Jinyong Lee \\ Modem\&Connectivity Team, System LSI \\ Samsung electronics \\ Yongin, Gyeonggi-do, Korea \\ jiny4509.lee@samsung.com
}

\author{
Jingu Lee, Youngseh Kim, Kanghoon Kim, Younglok Kim \\ Department of Electrical Engineering \\ Sogang University \\ Seoul, Korea \\ jingu8562@sogang.ac.kr, kyskim@sogang.ac.kr, \\ wsockkh@sogang.ac.kr, ylkim@sogang.ac.kr
}

\begin{abstract}
The FMCW radar estimates the relative speed and the distance of moving targets from the frequency spectrum of received beat signal. The radar systems based on the Doppler processing measure the appropriate information from discrete frequency spectrum, and hence the accuracy depends on the sampling rate as well as the number of samples. In this paper, we propose the frequency offset estimation method based on the kernel function analysis. The proposed method can compensate the errors by using the fine beat frequency obtained from the accurate offset values. It provides the high accuracy with the less number of samples, so the system has lower computational complexity and higher speed processing compared with conventional methods.
\end{abstract}

Keywords-FMCW; Doppler radar; automotive application; kernel function; frequency offset

\section{INTRODUCTION}

The FMCW radar is effectively used to measure the distance and the velocity of the moving objects. The $77 \mathrm{GHz}$ millimeter wave band is used for automotive applications [1] and many countries adopt this band through ITU recommendations [2].

In the FMCW radar systems, the frequency spectrum of received beat signals is analyzed to determine the existence of targets and also to obtain the information such as the distance and the relative speed of moving targets [3]. The CFAR algorithms [4,5] are developed to recognize the target by removing the clutters. After the CFAR detection process, the distance and the relative speed of target can be computed from measured beat frequency. Since the most FMCW radar system uses discrete Fourier transform (DFT) to obtain beat frequency, the discrete beat frequency must have the frequency offset which degrades the detection performance. The frequency offset is determined by sampling frequency $f_{s}$ and total number of samples $N$.

Many literatures have been proposed to improve the accuracy of radar systems. The bandwidth extrapolation (BWE), relaxation (RELAX) and compressive sensing (CS) estimation show the enhanced performance compared to the range Doppler method based on DFT [6]. The fine frequency estimation method proposed in [7] also can improve the resolution in discrete frequency domain, but it has drawback in computational complexity. Here, we propose a simple frequency offset estimation algorithm, which is based on the kernel function analysis [8].
This paper is organized as follows. The signal modeling is introduced in section 2. Then, the new frequency offset estimation method is proposed in section 3 . In section 4 , the performance of new method is evaluated by the simulations. The conclusive remarks are in section 5 .

\section{FMCW RADAR SIGNAL MODELING}

The FMCW radar system for automotive applications usually uses the triangular linear frequency modulation (LFM). The transmit signals are classified by the up chirp signal $s_{t x, u p}(t)$ and the down chirp signal $s_{t x, d n}(t)$, which are given by the following equations[9].

$$
\begin{aligned}
& s_{t x, u p}(t)=\exp \left\{j 2 \pi\left(f_{c} t+\alpha t^{2}\right)\right\} \\
& s_{t x, \text { dn }}(t)=\exp \left\{j 2 \pi\left(\left(f_{c}+\Delta F\right) t-\alpha t^{2}\right)\right\}
\end{aligned}
$$

Here, the frequency sweep rate, $\alpha$, is the ratio of bandwidth (BW) to pulse repetition interval (PRI). The received signal reflected from the moving target includes Doppler shift $f_{d}$ and time delay $\tau$, and received up chirp and down chirp signals are written by

$$
\begin{gathered}
s_{r x, u p}(t)=\exp \left\{j 2 \pi\left(\left(f_{c}+f_{d}\right)(t-\tau)+\alpha(t-\tau)^{2}\right)\right\} \\
=s_{t x, u p}(t) \cdot \exp \left\{j 2 \pi \left(\left(f_{d}-2 \alpha \tau\right) t\right.\right. \\
\left.\left.+\left(\alpha \tau-f_{c}-f_{d}\right) \tau\right)\right\}
\end{gathered}
$$

$$
\begin{gathered}
s_{r x, d n}(t)=\exp \left\{j 2 \pi\left(\left(f_{c}+\Delta F+f_{d}\right)(t-\tau)-\alpha(t-\tau)^{2}\right)\right\} \\
=s_{t x, d n}(t) \cdot \exp \left\{j 2 \pi \left(\left(f_{d}+2 \alpha \tau\right) t\right.\right. \\
\left.\left.-\left(\alpha \tau+f_{c}+\Delta F+f_{d}\right) \tau\right)\right\}
\end{gathered}
$$

After removing the reference signal from the received signal, the beat frequencies of the up chirp and down chirp signals are represented by

$$
\begin{aligned}
& f_{\text {beat,up }}=f_{d}-2 \alpha \tau \\
& f_{\text {beat }, d n}=f_{d}+2 \alpha \tau
\end{aligned}
$$


Now, the distance $R$ and relative speed $v$ can be obtained by

$$
\begin{aligned}
& R=\frac{c}{8 \alpha}\left(f_{\text {beat }, \text { dn }}-f_{\text {beat }, \text { up }}\right) \\
& v=\frac{\lambda}{2}\left(f_{\text {beat }, d n}+f_{\text {beat }, \text { up }}\right)
\end{aligned}
$$

Since the beat frequency is estimated at the discrete frequency domain, its accuracy is determined by the sampling rate as well as the number of samples. The maximum offset error is proportional to the system accuracy and it can be derived as

$$
\Delta f=\frac{f_{s}}{N} .
$$

\section{FREQUENCY OfFSET ESTIMATION METHOD}

The beat frequency of sampled signal causes interference to adjacent frequency index. And the effect of interference is determined by the offset of discrete beat frequency.

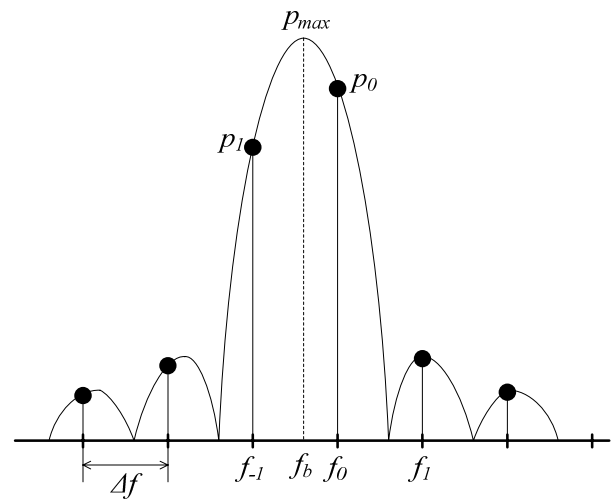

Figure 1. Ideal frequency spectrum plot by rectangular window.

Fig. 1 shows the frequency distribution of the sampled beat signal. The frequency distribution of window function can be expressed in kernel function, and the kernel function of rectangular window is sinc function. If magnitude $p_{0}$ is biggest magnitude and $p_{1}$ is the second largest magnitude, then they are described by

$$
\begin{aligned}
& p_{0}=p_{\text {max }} \cdot \operatorname{sinc}\left(e r r_{b f}\right) \\
& p_{1}=p_{\text {max }} \cdot \operatorname{sinc}\left(e r r_{b f}-1\right)
\end{aligned}
$$

Here, $p_{\max }$ is the maximum magnitude of frequency distribution, and the beat frequency error is assumed to be defined by

$$
\operatorname{err}_{b f}=\frac{N \cdot\left(\left|f_{0}-f_{\text {beat }}\right|\right)}{2 \pi}, \quad 0 \leq \operatorname{err}_{b f} \leq 0.5
$$

The rate of $p_{1}$ and $p_{0}$ can be written by follow equation

$$
\begin{aligned}
p_{\text {rate }} & =\frac{p_{1}}{p_{0}}=\frac{\operatorname{sinc}\left(e r r_{b f}-1\right)}{\operatorname{sinc}\left(e r r_{b f}\right)} \\
& =\frac{\sin \left(\pi \cdot e r r_{b f}-\pi\right) \cdot \pi \cdot \operatorname{err}_{b f}}{\sin \left(\pi \cdot \operatorname{err}_{b f}\right) \cdot \pi \cdot\left(\operatorname{err}_{b f}-1\right)}
\end{aligned}
$$

$$
=\frac{-e r r_{b f}}{e r r_{b f}-1}
$$

Hence, the beat error can be computed from $p_{\text {rate }}$ as

$$
\operatorname{err}_{b f}=\frac{p_{\text {rate }}}{p_{\text {rate }}+1}
$$

and we can obtain the compensated beat frequency $f_{b}$

$$
f_{b}=f_{0}-\frac{2 \pi \cdot e r r_{b f}}{N}
$$

The windowing are applied to CFAR detection performance for FMCW radar system. For automotive application system, the Hamming window provides good CFAR detection performance [10], and hence we focus on Hamming window and its kernel function. The Hamming window function $w_{h m}[n]$ is represented by

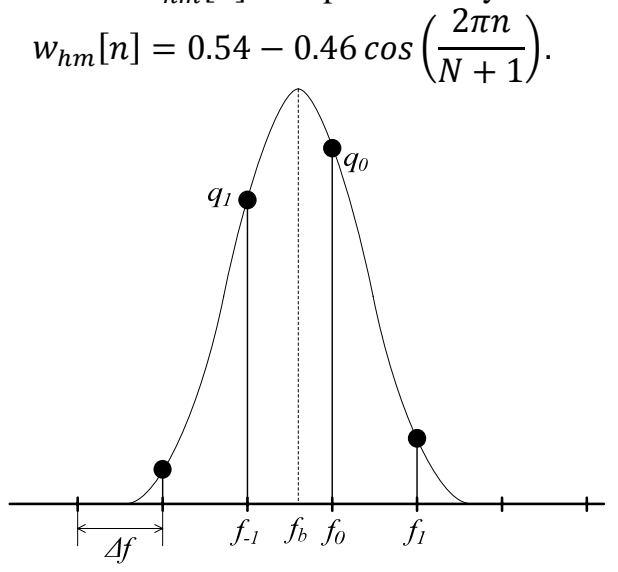

Figure 2. Ideal frequency spectrum plot by Hamming window

Fig. 2 shows the magnitude distribution of Hamming window in frequency domain, and the magnitude can be expressed in kernel function $W_{h m}(\theta)$ by [8]

$$
\begin{aligned}
W_{h m}(\theta) & =0.54 W_{r}(\theta)-0.23 W_{r}\left(\theta-\frac{2 \pi}{N-1}\right) \\
& -0.23 W_{r}\left(\theta+\frac{2 \pi}{N-1}\right)
\end{aligned}
$$

where $W_{r}(\theta)$ is the Fourier transform of rectangular window, which is given by

$$
W_{r}(\theta)=\frac{\sin (0.5 \theta N)}{\sin (0.5 \theta)} e^{-j 0.5 \theta(N-1)}
$$

As like the sinc function case, when $q_{0}$ and $q_{1}$ are the first and second biggest samples, the rate of magnitudes $q_{1}$ and $q_{0}$ can be represented by

$$
q_{\text {rate }}=\frac{q_{1}}{q_{0}}=\frac{W_{h m}\left(\frac{2 \pi\left(e r r_{b f}-1\right)}{N}\right)}{W_{h m}\left(\frac{2 \pi \cdot e r r_{b f}}{N}\right)}
$$




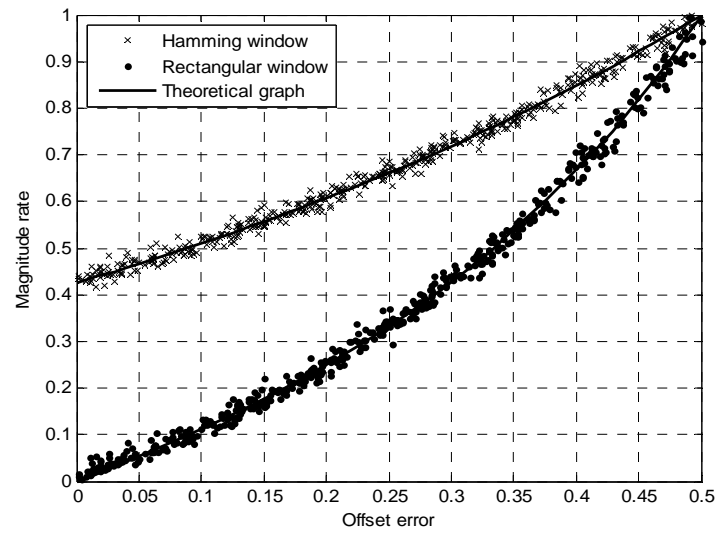

Figure 3. Offset error estimation comparison of Hamming and rectangular window

Fig. 3 shows the estimated offset error from the rate of magnitude. The rectangular window and the Hamming window cases are simulated with $0 \mathrm{~dB}$ SNR, and each result has come close to the measured offset errors. When the rectangular window is applied, the $\operatorname{err}_{b f}$ computation from $p_{\text {rate }}$ is easy, but in case of Hamming window, there is no closed form to obtain the inverse of kernel function. Hence, the look up table (LUT) is proposed to be used to get $\operatorname{err}_{b f}$ from Hamming function.

\section{Performance eVAluation}

The performance of proposed method is evaluated by computer simulations for automotive application environment [2] where the SNR is relatively higher. The system parameters for simulations are summarized in Table 1 . To obtain $\mathrm{err}_{b f}$ from kernel function of Hamming window, LUT of size 5,000 is used.

The $\operatorname{err}_{b f}$ using proposed offset estimation method can compensate the errors of measured values of moving target. And the accuracy of compensated values is proportional to SNR. Fig. 4 shows the average error of measured values with respect to the SNR for conventional method and proposed method with various DFT sizes. While the conventional system has error floor which are determined by the number of samples and sampling frequency, the proposed method provides the higher accuracy with the higher SNR. Also this method can obtain required accuracy with only smaller number of samples, and hence it can reduce the total computation complexity of system. The traditional method has same average errors regardless of window, but proposed method has better performance with Hamming window than rectangular window.
TABLE I. FMCW RADAR SYSTEM PARAMETERS

\begin{tabular}{cc}
\hline Parameter & Value \\
\hline Carrier frequency $\left(f_{c}\right)$ & Carrier freq $: 77 \mathrm{GHz}$ \\
Sweep time & $3 \mathrm{msec}$ \\
Sampling frequency & $369 \mathrm{khz}$ \\
Bandwidth & $150 \mathrm{MHz}$ \\
the frequency sweep rate $(\alpha)$ & $5 \times 10^{10}$ \\
FFT size $(\mathrm{N})$ & 1,024 \\
\hline
\end{tabular}

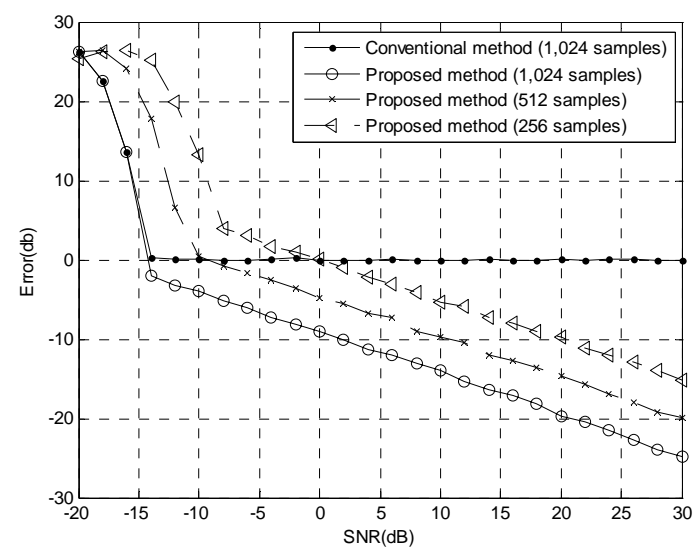

(a) Rectangular window

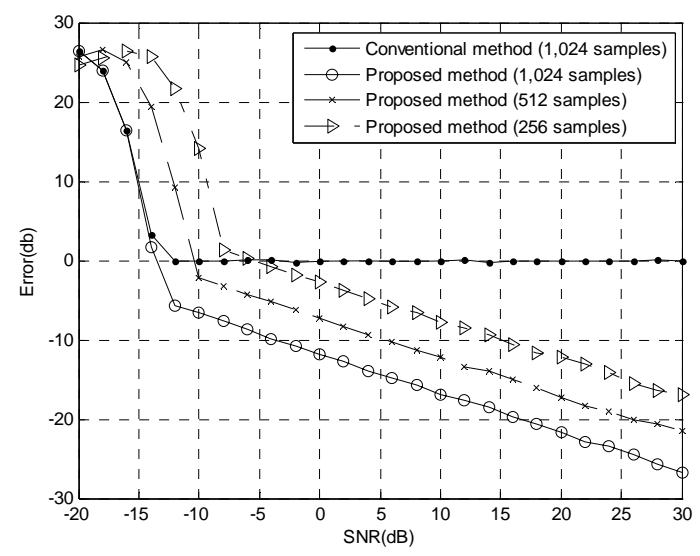

(b) Hamming window

Figure 4. The mean error of measured relative speed and distance for moving targets

\section{CONCLUSION}

The new simple estimation method for beat frequency offset is proposed to improve the accuracy of FMCW radar system. While the resolution of measured values is determined by sampling frequency and number of samples, the proposed method increases the accuracy of measured values without changing the sampling frequency or the number of sample. It decreases the errors with the small computational complexity. So, it can be applied to various radar systems based on Doppler processing. 


\section{ACKNOWLEDGMENT}

This work was supported by Research Program [B551179-12-07-00] funded by the Korea Research Council for ISTK(Industrial Science \& Technology) and the MKE(The Ministry of Knowledge Economy), Korea, under the CITRC(Convergence Information Technology Research Center) Support Program[NIPA-2012-H0401-12-2006] supervised by the NIPA(National IT Industry Promotion Agency) and the Sogang University Research Grant of 2012[201214004.01].

\section{REFERENCES}

[1] T. Mitomo et al., "A $77 \mathrm{GHz} 90 \mathrm{~nm}$ CMOS Transceiver for FMCW Radar Applications,” IEEE Journal of Solid State Circuits, vol. 45, no. 4, pp. 928-937, April 2010

[2] ITU-R Recommendation, "Millimeter wave radio communication systems for intelligent transport system applications,” M.1452-1, Oct. 2009
[3] A. G. Stove, "Linear FMCW radar techniques," Proc. IEE Proceedings-F, vol. 139, pp.343, 1992

[4] M. Skolnik, Radar handbook, 3rd Edition, New York, McGraw-Hill, 2008

[5] M. A. Richards, Fundamentals of radar signal processing, New York, McGraw-Hill, 2005

[6] L. Zhang, M. Xing, C. Qiu, J. Li, J. Sheng, Y. Li and Z. Bao, "Resolution Enhancement for Inversed Synthetic Aperture Radar Imaging Under Low SNR via Improved Compressive Sensing,” IEEE Trans. Geosci. Remote Sens., vol. 48, pp. 3,824-3,838, Oct. 2010

[7] C. Canda, "A Method For Fine Resolution Frequency Estimation From Three DFT Samples,” IEEE Signal Processing Letters, vol 18, pp. 351 - 354, June 2011

[8] B. Porat, A Course in Digitial Signal Processing, John Wiley and Sons, Oct. 1996

[9] A. Meta, P. Hoogeboom, and L. P. Ligthart, "Signal processing for FMCW SAR,” IEEE Trans. Geosci. Remote Sens., vol. 45, no. 11, pp. 3519-3532, 2007

[10] H. Rohling, "Radar CFAR thresholding in clutter and multiple target situations," IEEE Trans. on Aerospace and Electronic Systems, vol. 19, pp. 608-621, July 1983 